\title{
Multi-coil Magnetic Resonance imaging reconstruction with a Markov Random Field Prior
}

\author{
Marko Panića $^{\mathrm{a}}$,Jan Aelterman ${ }^{\mathrm{b}}$, Vladimir Crnojevića ${ }^{\mathrm{a}}$, and Aleksandra Pižurica ${ }^{\mathrm{b}}$

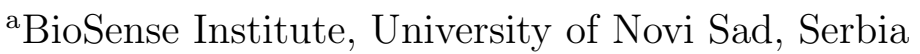 \\ bTELIN-IPI, Ghent University - imec, Ghent, Belgium
}

\begin{abstract}
Recent improvements in reconstructing Magnetic Resonance Images (MRI) from partial data have been reported using spatial context modelling with Markov Random Field (MRF) priors. However, these algorithms have been developed only for magnitude images from single-coil measurements. In practice, most of the MRI images today are acquired using multi-coil data. In this paper, we extend our recent approach for MRI reconstruction with MRF priors to deal with multi-coil data i.e., to be applicable in parallel MRI (pMRI) settings. Instead of reconstructing images from different coils independently and then obtaining final image using sum of squares method, we consider recovery of MRI image using undersampled measurements from all coils jointly with their estimated sensitivity maps. The proposed method is derived from a maximum a posteriori probability (MAP) formulation of the reconstruction problem on which is then applied an alternating direction method of multipliers (ADMM). Therefore, an algorithms steps of the proposed method can be divided in two blocks. The first one contains few iterations (up to 10) of conjugate gradient (CG) method for obtaining a temporary image estimate from multi-coil measurements which although are undersampled significantly overlap at low frequencies. The second block contains steps which refer to applications of MRF regularizations on temporary image estimate obtained from the first block. This procedure is repeated through iterations until some stopping criterion is satisfied (e.g. number of iterations). Experimental results demonstrate the effectiveness of the proposed approach in comparison to some well-adopted methods for accelerated pMRI reconstruction from undersampled data.
\end{abstract}

Keywords: Parallel MRI (pMRI), Markov Random Field (MRF), Alternating direction method of multipliers $(\mathrm{ADMM})$

\section{INTRODUCTION}

Slow MRI acquisition is still a barrier in everyday clinical usage. Parallel imaging in MRI (pMRI) and using a compressed sensing theory in MRI (CS-MRI) are the two possible directions how this speed limitation can be overcome. In this work we combine both these techniques for the purpose of multi-coil MRI reconstruction starting from the method which already proved it potential in single coil reconstruction scenario. A matematical model for a single coil MRI acquisition is given in the following equation

$$
\mathbf{y}=\mathbf{A x}+\mathbf{n}
$$

where image $\mathbf{x} \in \mathbb{C}^{N}$ has to be recovered from a $k$-space measurements data $\mathbf{y} \in \mathbb{C}^{M}$ with the present of white Gaussian noise $\mathbf{n} \in \mathbb{C}^{M}$. ${ }^{1,2}$ Matrix $\mathbf{A} \in \mathbb{C}^{M \times N}$, where $M \ll N$, denotes the undersampled Fourier operator formed according to a predefined $k$-space undersampling scheme e.g. (radial, spiral or random). Estimation of image $\mathbf{x}$ is usually obtained as a solution of the following optimization problem

$$
\min _{\mathbf{x} \in \mathbb{C}^{N}} \frac{1}{2}\|\mathbf{A x}-\mathbf{y}\|_{2}^{2}+\tau \phi(\mathbf{x})
$$

Further author information:

Marko Panić: E-mail: panic@biosense.rs

Jan Aelterman: E-mail: Jan.Aelterman@ugent.be

Vladimir Crnojević: E-mail: crnojevic@uns.ac.rs

Aleksandra Pižurica: E-mail: Aleksandra.Pizurica@ugent.be 
where the $\frac{1}{2}\|\mathbf{A x}-\mathbf{y}\|_{2}^{2}$ is a data fidelity term, $\phi: \mathbb{C}^{N} \mapsto \mathbb{R} \cup\{-\infty,+\infty\}$ is a regularization function and parameter $\tau$ which denotes the amount of involved regularization. The pMRI reconstruction problem can be formulated in image domain (SENSE method from ${ }^{3}$ ) or in $k$-space domain (GRAPPA method from ${ }^{4}$ ). In this work we opt for the maximum likelihood (ML) formulation of the multi-coil reconstruction problem given as

$$
\min _{\mathbf{x} \in \mathbb{C}^{N}} \sum_{i=1}^{N_{c}}\left\|\mathbf{y}_{i}-\mathbf{F C}_{i} \mathbf{x}\right\|_{2}^{2}
$$

where matrix $\mathbf{F}$ represents Fourier transform and index $i$ indicates the considered coil data $\mathbf{y}_{i}$ and its coressponding spatial sensitivity $\mathbf{C}_{i}$. If we consider reconstruction from a single coil $(i=1)$ we can see that equation (3) simplifies to form similar to the data fidelity term in (2). The difference is in the presence of undersampling in Fourier domain denoted with operator A instead of F. This means that involving CS in pMRI (pMRI-CS) reconstruction through the sampling of coils data below the Nyquist rate demands some sort of regularization since now reconstruction problem becomes ill-posed. In this work we extend the single coil reconstruction methods from $^{5}$ with MRF regularization for the more general pMRI-CS problem formulation.

\section{PARALLEL IMAGING IN MRI}

In the pMRI reconstruction the multiple $k$-space data $\mathbf{y}_{i}$ obtained from different reciever antenna coils are utilized in order to recover the MR image. The method $\mathrm{SENSE}^{3}$ combines the aliased coil images $\mathbf{x}_{i}$, reconstructed using inverse discrete 2-dimensional Fourier transform (2D-IDFT) from each coil measurements $\mathbf{y}_{i}$ (or density corrected adjoint non-uniform Fourier transform in case of non-Cartesian $k$-space trajectories), and creates a composite MR image with the a priori knowledge of coils sensitivities. Although an important issue with SENSE method is estimation of the coil sensitivity profiles, it is to date the most widespread employed pMRI techinque offered by many companies. The issue with estimation of sensitivity profiels can be circumvented with GRAPPA method ${ }^{4}$ which doesn't require knowledge of the coil sensitivities. It reconstructs the missing $k$-space data using its adjacent neighbourhood in $k$-space from all coils. GRAPPA uses a kernel which defines how the missing $k$-space from the $i^{t h}$ coil data are interpolated using the acquired $k$-space data from all of the coils. It is learned from the low-frequency spectrum of the $k$-space data of every coil (so-called Auto Calibration Signal (ACS)). ${ }^{6}$ After interpolation of the missing $k$-space data an 2D-IDFT is employed to obtained a $\mathbf{x}_{i}$ coil images which are usually with the Sum of Squares (SoS) techique:

$$
\mathbf{x}=\sqrt{\sum_{i=1}^{N_{c}}\left|\mathbf{x}_{i}\right|^{2}}
$$

combined to reconstruct the composite MR image $\mathbf{x}$. An approach for pMRI reconstruction SPIRiT (iterative selfconsistent parallel imaging reconstruction) is coil-by-coil method (reconstruct each coil image separately and then combine them to obtained the composite MR image) based on GRAPPA method. It synthesis observed missing $k$-space point using acquired and reconstructed missing points in its neighborhood from all coils. It achieved better noise reduction and more accurate reconstruction compared to traditional GRAPPA-like approaches. A method which bridge the gap between SENSE and GRAPPA, called ESPIRiT uses eigenvalue decomposition in image space for computation of robust sensitivity maps. It combines advantages of SPIRiT and GRAPPA methods and restrict solution to a subspace span by coil sensitivity maps.

For the case when the sampling of coils data is below the Nyquist rate a problem of image reconstruction becomes ill-posed and demands some sort of regularization. A method COMPASS from ${ }^{7}$ expresses a multi-coil reconstruction problem as Basis Pursuit (BP) optimization problem with $\ell_{1}$ norm as regularization on image sparse representation. Authors in ${ }^{8,9}$ proposed method which also combines parallel imaging with partial Fourier acquistion named LORAKS (LOw-RAnk modelling of local K-Space neighborhoods). It takes into account the fact that many MRI images have limited spatial support and smoothly varying phase through the constraints in optimization framework. In the following we propose a new method for the pMRI-CS reconstruction problem, based on method from. ${ }^{5}$ We create joint framework for utilization of parallel imaging and compressed sensing in MRI and compare it with the state-of-the-art methods in the field. 


\section{PROPOSED METHOD}

Let us formulate the pMRI-CS problem as follows:

$$
\min _{\mathbf{x} \in \mathbb{C}^{N}} \phi(\mathbf{P x}) \quad \text { subject to } \sum_{i=1}^{N_{c}}\left\|\mathbf{y}_{i}-\mathbf{A C}_{i} \mathbf{x}\right\|_{2}^{2} \leq \epsilon
$$

where $\mathbf{P}$ stands for sparsyfying transform and $\epsilon \geq 0$ denotes a parameter which is usually related to the noise variance. Since the measurements from the coils $\mathbf{y}_{i}$ are undersampled with operator $\mathbf{A}$, some regularization is necessary in order to find a suitable solution. Problem formulation in (5) has very similar form to the problem formulation from. ${ }^{5}$ The difference is that we have measurements gathered from more than one coil, which usually have overlaping in the region of low frequency components. This means that regularization step in algorithm for solving (5), has to be applied on image $\mathbf{x}$ which is initially (temporary) reconstructed using all coil measurements. If we introduce notation for augmented measurement vector $\mathbf{y}_{a}=\left[\mathbf{y}_{1}^{T}, \mathbf{y}_{2}^{T} \ldots \mathbf{y}_{N_{c}}^{T}\right]^{T}$ and augmented vectorized image $\mathbf{x}_{a}=\mathbf{T x}$, where $\mathbf{T}$ is formed by stacking $N_{c}$ times row-wise the indentity matrix $\mathbf{I}_{N \times N}$, then we can reformulate problem from (5) as

$$
\min _{\mathbf{x} \in \mathbb{C}^{N}} \phi(\mathbf{P} \mathbf{x}) \quad \text { subject to }\left\|\mathbf{y}_{a}-\mathbf{A}_{B} \mathbf{C}_{B} \mathbf{x}_{a}\right\|_{2}^{2} \leq \epsilon
$$

where $\mathbf{A}_{B}$ consists of repeated $\mathbf{A}$ along the diagonal while $\mathbf{C}_{B}$ is formed by stacking coil sensitivity maps $\mathbf{C}_{i}$ along diagonal. A constrained optimization problem in (6) can be transformed to its unconstrained form, with a usage of an indicator function, ${ }^{5}$ which is equivialent to maximum a posteriori probability (MAP) formulation for estimation of original image $\mathbf{x}$. Obtained unconstrained form of the problem in (6) is then solved by application of alternating direction method of multipliers (ADMM). Derived ADMM algorithm steps can be divided in two blocks the first one which refers to obtaining temporary image estimate from all coils measurements using few iterations (up to 10) of conjugate gradient (CG) and the second block which contains steps for application of MRF regularizations.

\section{EXPERIMENTAL EVALUATION}

In the first experiment we compared our proposed method with the COMPASS ${ }^{7}$ method on a saggital MRI slice acquired using measurements from 4 coils with $12 \%$ of the Nyquist sampling rate on a $k$-space spiral trajectory. This leads to an ill-posed problem since the overall number of measurements is less than the Nyquist sampling rate. The results, shown in Fig. 1, demonstrate the advantage of our regularization based on MRF prior compared to the $\ell_{1}$ norm regularization in the multi-coil reconstruction scenario. Although both methods used the same coil sensitivity maps, the proposed approach yields an improvement in the peak signal to noise ration (PSNR) of more than $6 \mathrm{~dB}$. The reconstructed image is much sharper and has preserved much more original details, as it also evident from the diference image.

The second experiment was evaluated on data publicly available on http://www.eecs. berkeley.edu/ mlustig which is used in the experimental evaluation of methods ESPIRIT ${ }^{10}$ and SPIRIT. ${ }^{11}$ The lower sampling rate is obtained by decimating the data with acceleration factor greater than 1 . We use measurements undersampled with acceleration factor 3 from 4 virtual coils obtained from 8 coils using SVD coil compression technique. This experimental setup is the same as the one used in. ${ }^{10,11}$ In the proposed method the coil sensitivity maps are estimated from undersampled measurements using approach from. ${ }^{12}$ From the presented results in Fig. 2 we see that proposed method outperforms all compared methods. An image artifacts caused by undersampling trajectory are better suppresed in image reconstructed by proposed method than in images recovered by compared methods.

In the last experiment we compare our method with the P-LORAKS and SENSE-LORAKS methods. ${ }^{8,9}$ We use four-channel $\mathrm{T} 1$ weighted brain image from ${ }^{9}$ with provided coil sensitivity profiles. Reconstruction is conducted using $14 \%$ of measurements from each coil sampled using two different trajectories random and uniform. A golden standard image is created using all measurements from four coils with the SoS techique. Achieved reconstruction performances are given in Table. 1. Reconstruction obtained with the proposed method outperformed compared methods. Visual comparison is presented in Fig. 3 and in Fig. 4 for the uniform and 

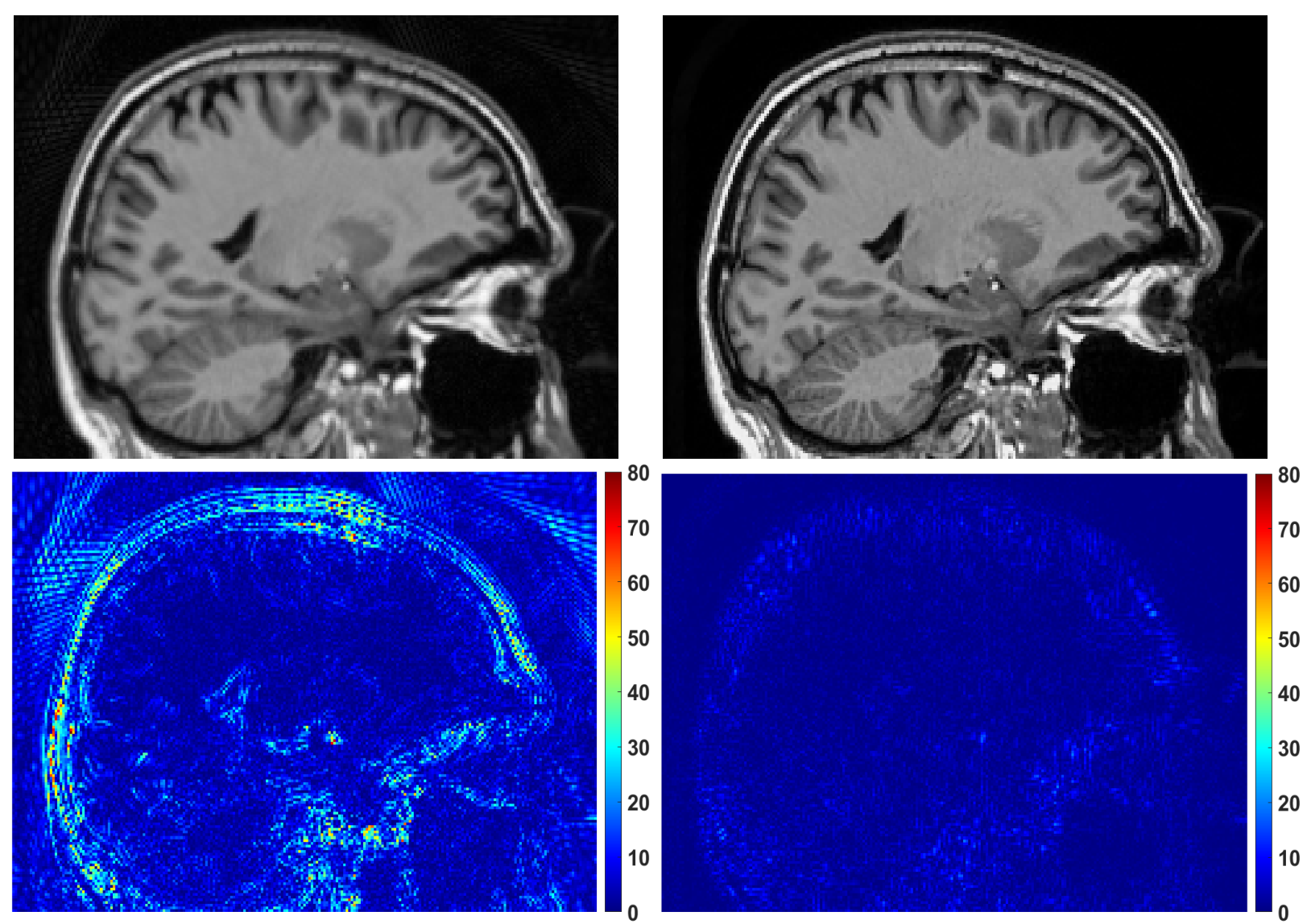

Figure 1. Reconstruction of sagittal slice from 4 coils with $12 \%$ of measurements per coil sampled with spiral trajectory. First row: Reconstructed images with $\operatorname{COMPAS}^{7}(27.75 \mathrm{~dB})$ and proposed method $(35.42 \mathrm{~dB})$ method respectively. Second row: Coresponding reconstruction errors.

random sampling trajectory respectively. We can see in reconstructed image with the proposed methods a much smaller amount of noise present compared to the reconstructions obtained by P-LORAKS and SENSE-LORAKS.

Table 1. Reconstruction of image from ${ }^{9}$ using undersampled measurements obtained from 4 coils with uniform / random trajectory. A comparison of P-LORACS and SENSE-LORAKS methods with the proposed one is given in terms of structure similarity measure (SSIM) and normalized root-mean-squared-error (NRMSE).

\begin{tabular}{|c|c|c|}
\hline Methods & SSIM & NRMSE \\
\hline P-LORAKS & $0.88 / 0.92$ & $0.07 / 0.06$ \\
\hline SENSE-LORAKS & $0.83 / 0.87$ & $0.08 / 0.07$ \\
\hline Proposed method & $0.95 / 0.96$ & $0.04 / 0.03$ \\
\hline
\end{tabular}

\section{CONCLUSION}

In this work we extend our recent approach for MRF based MRI reconstruction to deal with more general multicoil acquisitions. The extension is based on (MAP) signal estimation from undersampled multi-coil measurements which is conducted using ADMM. The derived algorithm contains block of steps regarding temporary MRI image estimate and block of steps which refer to MRF regularization which alternate one after the other through 

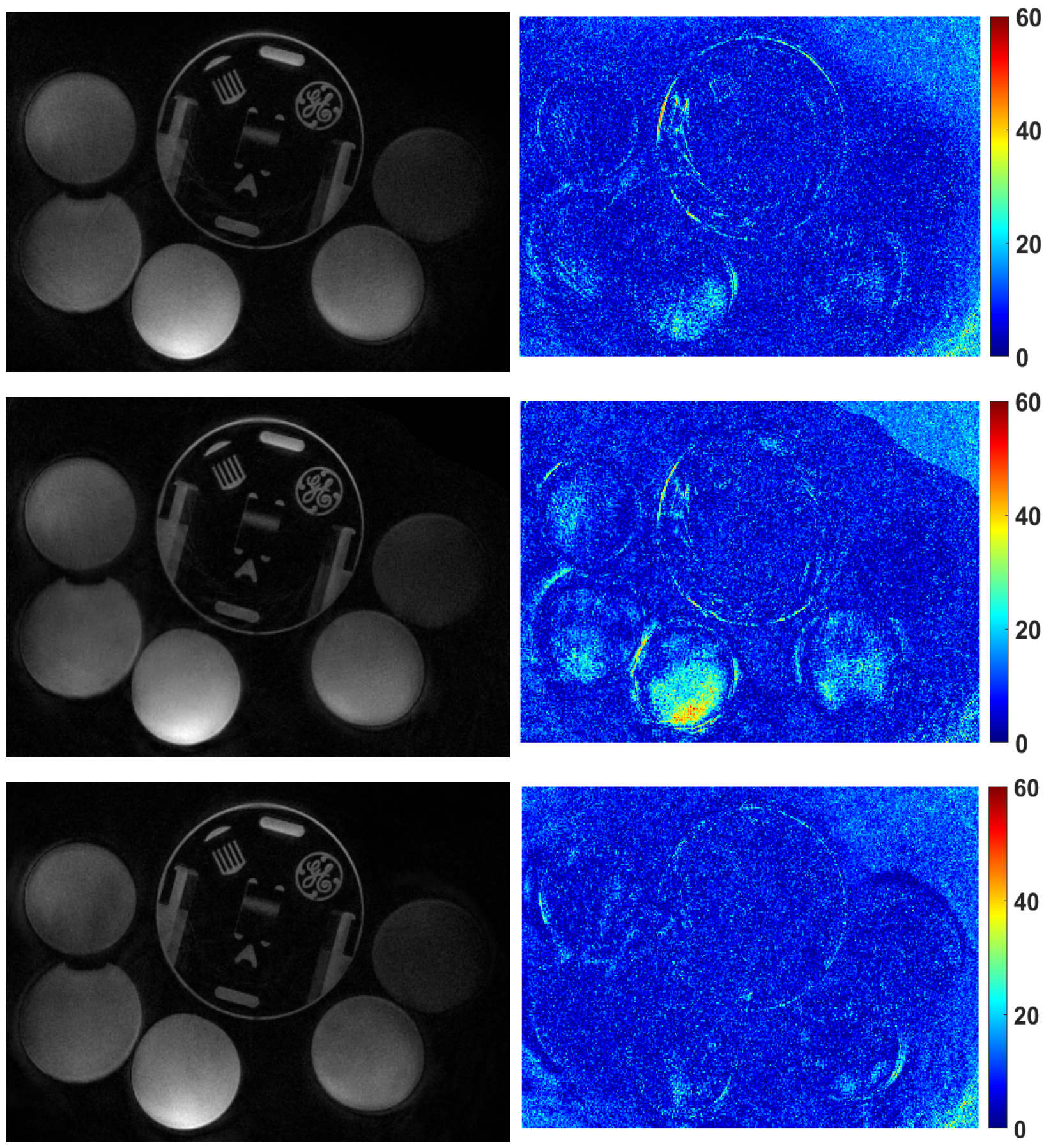

Figure 2. Reconstruction of phantom test image using measurements sampled with acceleration factor 3 from 4 virtual coils. First column: Reconstructed images with SPIRIT (32.22 dB), ESPIRIT (31.32 dB) and proposed method (32.64 dB) respectively. Second column: Coresponding reconstruction errors.

iterations until some stoping criterion is satisfied. From the experemental results we see that proposed method outperforms some well-adopted method in multi-coil reconstruction scenario. 

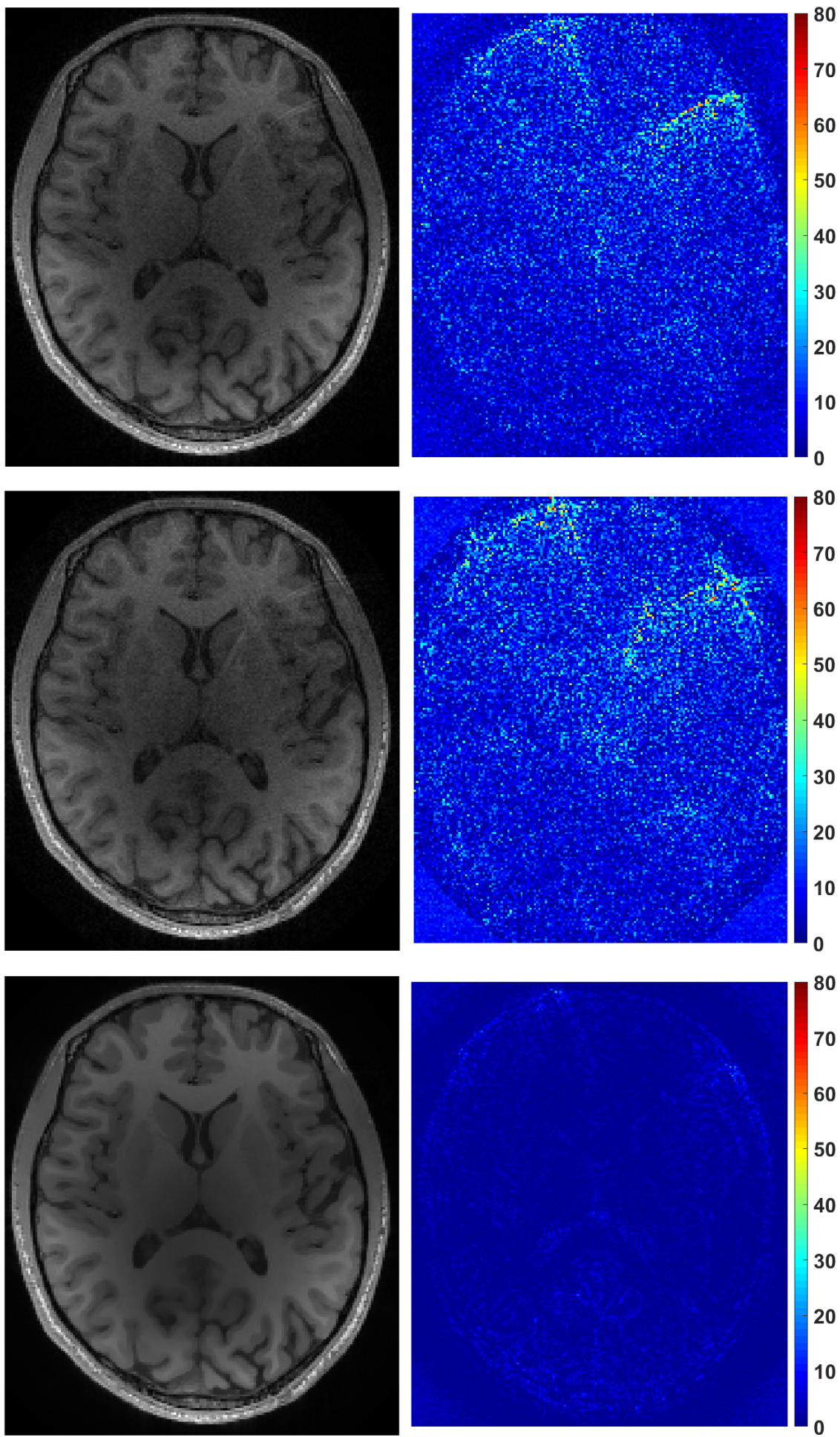

Figure 3. Reconstruction of T1 weighted brain image from ${ }^{9}$ form uniformly sampled measurements from 4 coils. First column: Reconstructed images with P-LORAKS, SENSE-LORAKS and proposed method respectively. Second column: Coresponding reconstruction errors. 

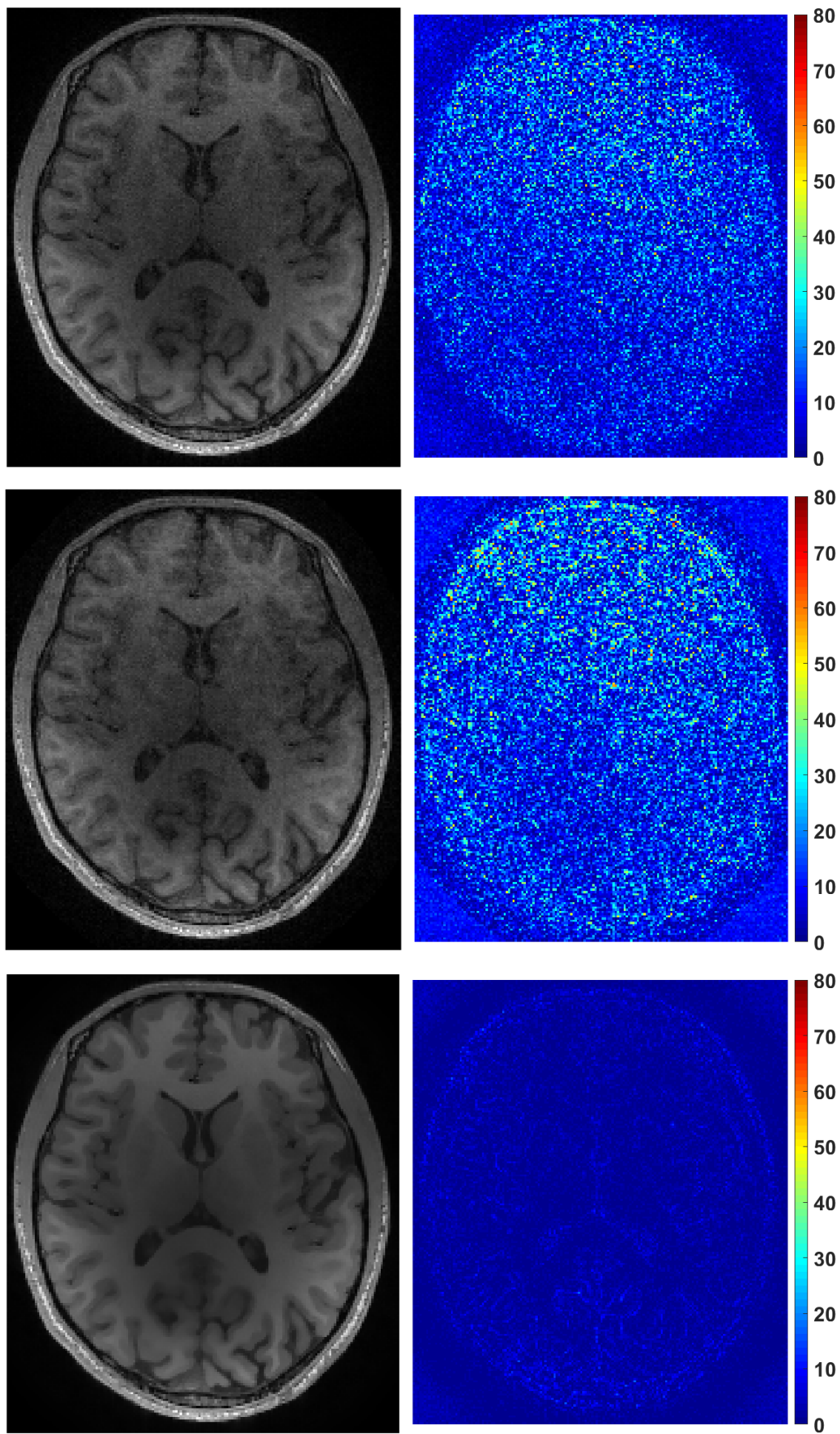

Figure 4. Reconstruction of T1 weighted brain image from ${ }^{9}$ form randomly sampled measurements from 4 coils. First column: Reconstructed images with P-LORAKS, SENSE-LORAKS and proposed method respectively. Second column: Coresponding reconstruction errors.

\section{ACKNOWLEDGMENTS}

This research has been supported by the H2020 project ANTARES No664387. We are grateful to Prof. Dr. Karel Deblaere from the Radiology Department of UZ Gent hospital for providing the data. 


\section{REFERENCES}

[1] Lustig, M., Donoho, D., and Pauly, J. M., "Sparse MRI: The application of compressed sensing for rapid MR imaging," Magnetic Resonance in Medicine 58(6), 1182-1195 (2007).

[2] Lustig, M., Donoho, D. L., Santos, J. M., and Pauly, J. M., "Compressed sensing MRI," IEEE Signal Process. Mag. 25(2), 72-82 (2008).

[3] Pruessmann, K. P., Weiger, M., Scheidegger, M. B., and Boesiger, P., "Sense: sensitivity encoding for fast mri," Magnetic resonance in medicine 42(5), 952-962 (1999).

[4] Blaimer, M., Breuer, F., Mueller, M., Heidemann, R. M., Griswold, M. A., and Jakob, P. M., "Smash, sense, pils, grappa: how to choose the optimal method," Topics in Magnetic Resonance Imaging 15(4), 223-236 (2004).

[5] Panić, M., Aelterman, J., Crnojević, V., and Pižurica, A., "Sparse recovery in magnetic resonance imaging with a markov random field prior," IEEE transactions on medical imaging 36(10), 2104-2115 (2017).

[6] Griswold, M. A., Jakob, P. M., Heidemann, R. M., Nittka, M., Jellus, V., Wang, J., Kiefer, B., and Haase, A., "Generalized autocalibrating partially parallel acquisitions (grappa)," Magnetic Resonance in Medicine: An Official Journal of the International Society for Magnetic Resonance in Medicine 47(6), 1202-1210 (2002).

[7] Aelterman, J., Luong, H. Q., Goossens, B., Pižurica, A., and Philips, W., "Compass: a joint framework for parallel imaging and compressive sensing in mri," in [Image Processing (ICIP), 2010 17th IEEE International Conference on], 1653-1656, IEEE (2010).

[8] Haldar, J. P. and Zhuo, J., "P-loraks: Low-rank modeling of local k-space neighborhoods with parallel imaging data," Magnetic resonance in medicine 75(4), 1499-1514 (2016).

[9] Kim, T. H., Setsompop, K., and Haldar, J. P., "Loraks makes better sense: Phase-constrained partial fourier sense reconstruction without phase calibration," Magnetic resonance in medicine 77(3), 1021-1035 (2017).

[10] Uecker, M., Lai, P., Murphy, M. J., Virtue, P., Elad, M., Pauly, J. M., Vasanawala, S. S., and Lustig, M., "Espirit - an eigenvalue approach to autocalibrating parallel mri: where sense meets grappa," Magnetic resonance in medicine $\mathbf{7 1 ( 3 ) , 9 9 0 - 1 0 0 1 ~ ( 2 0 1 4 ) . ~}$

[11] Lustig, M. and Pauly, J. M., "Spirit: iterative self-consistent parallel imaging reconstruction from arbitrary k-space," Magnetic resonance in medicine 64(2), 457-471 (2010).

[12] Aelterman, J., Naeyaert, M., Gutierrez, S., Luong, H., Goossens, B., Pižurica, A., and Philips, W., "Automatic high-bandwidth calibration and reconstruction of arbitrarily sampled parallel mri," PloS one $\mathbf{9}(6)$, e98937 (2014). 